\title{
CONCENTRACIÓN DE MICRONUTRIMENTOS Y CRECIMIENTO DE RAÍZ EN VARIEDADES DE ARROZ EXPUESTAS A ALUMINIO
}

\section{MICRONUTRIENT CONCENTRATION AND ROOT GROWTH IN RICE VARIETIES EXPOSED TO ALUMINUM}

\author{
Fernando C. Gómez-Merino ${ }^{1 \star}$, Libia I. Trejo-Téllez ${ }^{2}$ y Tania Marín-Garza ${ }^{2}$
}

\author{
${ }^{1}$ Campus Córdoba, Colegio de Postgraduados. Km 348 Carretera Córdoba-Veracruz, Congregación Manuel León. 94946, Amatlán de los Reyes, Veracruz, México. \\ ${ }^{2}$ Campus Montecillo, Colegio de Postgraduados. Km 36.5 Carretera México-Texcoco, Montecillo. 56230, Texcoco, Estado de México, México. \\ ${ }^{*}$ Autor para correspondencia (fernandg@colpos.mx)
}

\section{RESUMEN}

El aluminio $\left(\mathrm{Al}^{3+}\right)$ es uno de los mayores factores limitantes de la producción agrícola en suelos ácidos con $\mathrm{pH}$ menor a 5 en las regiones tropicales del mundo. El arroz (Oryza sativa L.) ha mostrado ser tolerante a este factor de estrés, con respuestas diferenciales entre genotipos. Se evaluó el efecto de tres niveles de $\mathrm{Al}(0,200$ y $400 \mu \mathrm{M}$ $\mathrm{AlCl}_{3} 6 \mathrm{H}_{2} \mathrm{O}$ ) en la solución nutritiva sobre la concentración foliar de micronutrimentos $(\mathrm{Cu}, \mathrm{Fe}, \mathrm{Mn}$ y $\mathrm{Zn})$ y longitud de raíz en cuatro genotipos de arroz ('Huimanguillo, 'Koshihikari', 'Temporalero' y 'Tres Ríos'), en condiciones de invernadero, en un experimento factorial. $\mathrm{La}$ interacción de los factores evaluados (variedad $\mathrm{x}$ concentración de $\mathrm{Al}$ ) mostró efectos significativos en la concentración de los cuatro nutrimentos y en la longitud de raíz. En general, a medida que se aumentó la concentración de Al en la solución nutritiva, hubo un aumentó en las concentraciones de $\mathrm{Cu}$ y $\mathrm{Fe}$ en todas las variedades, mientras que las concentraciones de Mn y $\mathrm{Zn}$ disminuyeron. La mayor disminución de las concentraciones nutrimentales se observó en las variedades 'Huimanguillo', 'Koshihikari' y 'Tres Ríos', en tanto que la variedad 'Temporalero' resultó menos afectada. Respecto a la longitud de raíz, los mayores niveles de $\mathrm{Al}$ en solución redujeron significativamente a esta variable, y las variedades más afectadas fueron 'Temporalero' y 'Tres Ríos'. Se concluye que el Al afecta diferencialmente los genotipos de arroz evaluados, y que este metal muestra sinergismo con $\mathrm{Cu}$ y Fe, y antagonismo con Mn y Zn.

Palabras clave: Oryza sativa, cobre, hierro, manganeso, zinc.

\section{SUMMARY}

Aluminum $\left(\mathrm{Al}^{3+}\right)$ is one of the most limiting factors of agricultural production in acid soils with $\mathrm{pH}$ lower than 5 in tropical regions worldwide. Rice (Oryza sativa $\mathrm{L}$.) has showed to be tolerant to this stress factor, and differences in tolerance responses to $\mathrm{Al}$ have been observed among genotypes. We evaluated the effect of three $\mathrm{Al}$ concentrations $\left(0,200\right.$ and $\left.400 \mu \mathrm{M} \mathrm{AlCl}_{3} 6 \mathrm{H}_{2} \mathrm{O}\right)$ in the nutrient solution on the foliar concentration of micronutrients $(\mathrm{Cu}, \mathrm{Fe}, \mathrm{Mn} \mathrm{y} \mathrm{Zn})$ and root length in four rice genotypes ('Huimanguillo', 'Koshihikari', 'Temporalero' and 'Tres Ríos') grown under greenhouse conditions, in a factorial experiment. Interaction between factors evaluated (variety x Al concentration) showed significant effects on the concentrations of the four micronutrients evaluated and on root length as well. In general, as $\mathrm{Al}$ concentration was raised in the nutrient solution, an increase of the $\mathrm{Cu}$ and $\mathrm{Fe}$ concentrations was evident in the four varieties, while $\mathrm{Mn}$ and $\mathrm{Zn}$ concentrations diminished. The highest reduction on micronutrient concentration was observed in the varieties 'Huimanguillo, 'Koshihikari' and 'Tres Ríos', whereas the variety 'Temporalero' was less affected. Concerning root length, the highest levels of $\mathrm{Al}$ in the nutrient solution significantly caused a reduction in this variable, and the varieties most affected were 'Temporalero' and 'Tres Ríos'. We conclude that $\mathrm{Al}$ differentially affects the rice genotypes evaluated, and that this metal shows diminished to $\mathrm{Cu}$ and $\mathrm{Fe}$, and antagonism to $\mathrm{Mn}$ and $\mathrm{Zn}$.

Index words: Oryza sativa, copper, iron, manganese, zinc.

\section{INTRODUCCIÓN}

En México, la toxicidad por aluminio (Al) es una de las mayores limitantes para la producción agrícola en suelos ácidos tropicales con $\mathrm{pH}$ menor a 5, y se estima que este problema afecta a más de 14 millones de hectáreas distribuidas principalmente en el sureste del país (Marín-Garza et al., 2010).

La forma iónica $\mathrm{Al}^{3+}$ se considera tóxica para la rizosfera, definida ésta como la capa de suelo que es afectada por el metabolismo radical (Berg et al., 2005). Por su parte, las formas de hidróxido de $\mathrm{Al}\left[\mathrm{Al}(\mathrm{OH})^{2+}, \mathrm{Al}(\mathrm{OH})_{2}{ }^{+}\right.$ y $\left.\mathrm{Al}(\mathrm{OH})_{4}^{-}\right]$, presentes en la solución entre 5 y $6.2 \mathrm{de} \mathrm{pH}$, han mostrado no ser tóxicas para las plantas (Trejo-Téllez y Gómez-Merino, 2007).

Los efectos tóxicos del $\mathrm{Al}^{3+}$ se relacionan con una rápida inhibición del crecimiento radical al acumularse en el apoplasto, la membrana plasmática y eventualmente en el citosol (Vardar y Ünal, 2007). Producción de especies reactivas de oxígeno (ROS), desbalance nutrimental, acumulación de calosa y pérdida de la homeostasis del calcio $\left(\mathrm{Ca}^{2+}\right)$ citosólico, son algunos de los efectos tóxicos del $\mathrm{Al}^{3+}$ una vez introducido en la célula (Silva, 2012).

La exudación de malato, de fosfato o ambos en raíces de arroz, puede funcionar como un mecanismo novedoso para quelatar $\mathrm{Al}^{3+}$ en el apoplasto de las células del ápice radical. La función primaria de los exudados radicales como mecanismo de tolerancia a $\mathrm{Al}$ es la exclusión del metal del ápice radical, pero esto no explica los altos niveles de tolerancia a 
Al observados en arroz (Oryza sativa L.). La evidencia más clara de este hecho es que la expresión ectópica del gen de tolerancia a $\mathrm{Al} A L M T 1$ de trigo (Triticum aestivum L.) en arroz, induce la exudación de malato pero no modifica la tolerancia a $\mathrm{Al}$ (Sasaki et al., 2004).

Sin embargo, cuando el gen ALMT1 es introducido en el genoma de cebada (Hordeum vulgare L.), una especie susceptible a $\mathrm{Al}$, se observa incremento de la tolerancia a este metal en más de $100 \%$ en comparación con plantas testigo no transformadas. Además, se han identificado loci de características cuantitativas (QTL) de tolerancia a Al muy cercanos a genes homólogos al gen ALMT1, lo que permite suponer que este conjunto de genes dan la mayor tolerancia a $\mathrm{Al}$ en arroz. Dado que la mayor parte del $\mathrm{Al}$ se acumula en el apoplasto en células radicales, la pared celular juega un papel determinante en la tolerancia a este metal observada en arroz, posiblemente a través de polisacáridos estructurales (Famoso et al., 2010).

En arroz, la tolerancia a Al varía entre genotipos. En general, los cultivares más tolerantes son de la subespecie japonica, en tanto que los de indica son más susceptibles al aluminio en solución (Watanabe y Okada, 2005). MarínGarza et al. (2010) mostraron que los cultivares 'Temporalero', 'Huimanguillo', 'Tres Ríos' y 'Koshihikari' dieron respuestas diferenciales al aluminio en la solución nutritiva, y el cultivar 'Tres Ríos' fue el más tolerante a $\mathrm{Al}$ porque no mostró daños en sus tejidos radicales y logró acumular menores niveles de $\mathrm{Al}$ y mayores niveles de $\mathrm{Mg}$ y $\mathrm{P}$ en hoja.

Los estudios del efecto de $\mathrm{Al}$ en la nutrición de cultivos, se han realizado principalmente en macronutrimentos, en especial P, K, Ca y Mg. Por tanto, en la presente investigación se estudió el efecto que ejerce la presencia de $\mathrm{Al}$ en la solución nutritiva en la acumulación de los micronutrimentos $\mathrm{Cu}, \mathrm{Fe}, \mathrm{Mn}$ y $\mathrm{Zn}$, y en el crecimiento radical en los cultivares de arroz 'Temporalero, 'Huimanguillo, 'Tres Ríos' y 'Koshihikari'.

\section{MATERIALES Y MÉTODOS}

\section{Condiciones de estudio}

La investigación se hizo en un invernadero tipo cenital de estructura metálica y plástico calibre 600 (transmitancia luminosa de 50 \%) ubicado en Montecillo, Texcoco, Estado de México (a $19^{\circ} 20^{\prime} \mathrm{N}, 98^{\circ} 53^{\prime} \mathrm{O}$, y altitud de $2250 \mathrm{~m}$ ).

\section{Material vegetal}

El estudio dio inicio con la germinación de semillas de cuatro variedades de arroz (las mexicanas 'Temporalero', 'Huimanguillo,' 'Tres Ríos' y la japonesa 'Koshihikari'), en cajas Petri sobre papel filtro humedecido con agua destilada. Ocho días después de la germinación, las plántulas fueron trasplantadas a charolas de plástico que contenían suelo esterilizado $(\mathrm{pH} 7.8$, conductividad eléctrica $1.09 \mathrm{dS}$ $\mathrm{m}^{-1}, \mathrm{~N} 0.05 \%$, P $59.53 \mathrm{mg} \mathrm{kg}^{-1}, \mathrm{~K}^{2} .38 \mathrm{cmol}_{(+)} \mathrm{kg}^{-1}$, capacidad de intercambio catiónico $15.26 \mathrm{cmol}_{(+)} \mathrm{kg}^{-1}$, textura franco arcillosa), donde permanecieron hasta alcanzar $35 \mathrm{~d}$ de edad y $8 \mathrm{~cm}$ de altura. Posteriormente, las plantas fueron extraídas del suelo, sus raíces se lavaron con agua destilada, y luego se trasplantaron a macetas de $3 \mathrm{~L}$ de capacidad que contenían la solución nutritiva de Steiner (a $20 \%$, con pH de 5.5), y mantenidas ahí por una semana como periodo de adaptación.

\section{Tratamientos y diseño experimental}

Los tratamientos de aluminio $(0,200$ y $400 \mu \mathrm{M} \mathrm{Al})$ fueron aplicados en un experimento con arreglo factorial $4 \mathrm{x}$ 3 , donde el factor 1 fue la variedad de arroz ('Temporalero', 'Huimanguillo, 'Tres Ríos' y 'Koshihikari'), y el factor 2 fue la concentración de $\mathrm{Al}^{3+}$. Los 12 tratamientos fueron distribuidos en un diseño experimental completamente al azar, en el que una cubeta de $3 \mathrm{~L}$ de capacidad con cuatro plantas fue considerada como unidad experimental, con tres cubetas por tratamiento.

\section{Solución nutritiva}

La solución nutritiva de Steiner (Steiner, 1984) a $20 \%$ usada para el crecimiento de las plantas contenía los siguientes iones (en mol $\mathrm{cm}^{-3}$ ): $2.4 \mathrm{de} \mathrm{NO}_{3}^{-} ; 0.2 \mathrm{de}_{2} \mathrm{PO}_{4}^{-}$; $1.4 \mathrm{de}_{4}^{-2} ; 1.4$ de $\mathrm{K}^{+} ; 1.8$ de $\mathrm{Ca}^{2+}$; y $0.8 \mathrm{de} \mathrm{Mg}^{2+}$. Adicionalmente se agregó una mezcla de micronutrimentos en las siguientes concentraciones $\left(\mathrm{mg} \mathrm{L}^{-1}\right): 1.6$ de $\mathrm{Mn} ; 0.11 \mathrm{de} \mathrm{Cu}$; 0.865 de B; 0.023 de $\mathrm{Zn}$; y 5.0 de Fe, en la que $\mathrm{Mn}$, $\mathrm{Cu}$ y $\mathrm{Zn}$ fueron suministrados en forma de sulfatos; $\mathrm{B}$ como $\mathrm{H}_{3} \mathrm{BO}_{3}$; y Fe como quelato (Fe-EDTA), según lo descrito por Steiner $y$ van Winden (1970). El aluminio fue suministrado como $\mathrm{AlCl}_{3} 6 \mathrm{H}_{2} \mathrm{O}$ y el pH de la solución fue ajustado a un valor de 4.2, para garantizar que el Al permaneciera en su forma iónica $\mathrm{Al}^{3+}$. La solución nutritiva fue renovada cada $48 \mathrm{~h}$, con un ajuste de pH a 4.2 cada $24 \mathrm{~h}$. Debido a que el arroz es una especie que se cultiva bajo condiciones de anoxia, la solución nutritiva no fue oxigenada.

\section{Variables medidas}

Concentración de micronutrimentos. Después de cuatro semanas de tratamiento con $\mathrm{Al}$, las plantas fueron cosechadas y las hojas fueron separadas y secadas para la determinación de las concentraciones de $\mathrm{Cu}, \mathrm{Fe}, \mathrm{Mn}$ y $\mathrm{Zn}$. Las muestras de material seco se sometieron a digestión con una mezcla de ácidos nítrico y perclórico (relación 2:1, v:v) (Alcántar y Sandoval, 1999). En los extractos obtenidos se 
cuantificaron las concentraciones de $\mathrm{Cu}, \mathrm{Fe}, \mathrm{Mn}, \mathrm{Zn}$ y Al con un equipo de espectrometría de emisión atómica de inducción por plasma acoplado (ICP-AES) marca VARIAN ${ }^{\circledR}$ modelo Liberty II (Mulgrave, Australia).

Tamaño y crecimiento de raíces. En las semanas 2 y 4 del experimento se midió longitud y diámetro de raíces (en $\mathrm{mm}$ ), y se tomaron fotografías para registrar su forma.

Análisis estadístico. Los datos obtenidos fueron analizados mediante el programa estadístico SAS (SAS Institute, 2011), con el procedimiento ANOVA y las medias fueron comparadas por la prueba de Tukey $(\mathrm{P} \leq 0.05)$.

\section{RESULTADOS Y DISCUSIÓN}

El análisis nutrimental demostró que la variedad, la concentración de $\mathrm{Al}$ y la interacción entre ambos factores ejercieron efectos significativos $(\mathrm{P} \leq 0.05)$ sobre la concentración de $\mathrm{Cu}, \mathrm{Fe}, \mathrm{Mn}$ y Zn en hojas de arroz (Cuadro 1). El factor variedad y la interacción entre factores de estudio tuvieron efectos significativos en la concentración de los micronutrimentos en estudio, mientras que el factor concentración de $\mathrm{Al}$ en la solución nutritiva tuvo efectos significativos sobre la concentración de $\mathrm{Cu}, \mathrm{Mn}$ y Zn, pero no para Fe.

El efecto de la interacción entre los factores medidos (variedad $\mathrm{x}$ concentración de $\mathrm{Al}$ ) sobre la concentración de $\mathrm{Cu}$, $\mathrm{Fe}, \mathrm{Mn}$ y $\mathrm{Zn}$ en hojas de arroz se muestra en el Cuadro 2. Se aprecia que la mayor concentración de $\mathrm{Cu}$ se obtuvo en la variedad 'Temporalero' tratada con $400 \mu \mathrm{M}$ Al. Esta misma variedad no mostró efecto de las interacciones entre los factores en estudio para la concentración de Fe, y lo mismo se observa en la variedad 'Tres Ríos'. Respecto a Zn, su concentración aumentó conforme se incrementó la concentración de $\mathrm{Al}$ en la solución en todas las variedades.

La concentración de $\mathrm{Cu}, \mathrm{Fe}, \mathrm{Mn}$ y $\mathrm{Zn}$ en hojas de arroz sometidas a tres concentraciones de $\mathrm{Al}$ en la solución nutririva por efecto del factor variedad se presenta en el Cuadro 3. Las variedades 'Koshihikari,' 'Tres Ríos' y 'Huimanguillo' acumularon menos $\mathrm{Zn}$ en sus hojas, comparadas con 'Temporalero', que mantuvo una concentración mayor de este elemento, y también de $\mathrm{Cu}$ y Mn. Respecto a Fe, la variedad 'Huimanguillo' presentó la menor concentración.

El efecto del factor concentración de Al en la solución nutritiva sobre las concentraciones foliares de $\mathrm{Cu}, \mathrm{Fe}, \mathrm{Mn}$ y $\mathrm{Zn}$ se muestra en el Cuadro 4. Las mayores concentraciones de $\mathrm{Cu}$ y Fe se presentaron en plantas expuestas a los mayores niveles de Al en solución ( $400 \mu \mathrm{M} \mathrm{Al})$. Las concentraciones de $\mathrm{Mn}$ y $\mathrm{Zn}$ mostraron una respuesta opuesta a $\mathrm{Cu}$ y $\mathrm{Fe}$, dado que la mayor concentración de Mn y Zn se observó en plantas testigo ( $\sin \mathrm{Al}$ en la solución nutritiva), en tanto que su menor concentración se tuvo en plantas tratadas con el nivel más alto de $\mathrm{Al}(400 \mu \mathrm{M} \mathrm{Al})$, lo que demuestra un efecto antagónico de $\mathrm{Al}$ sobre $\mathrm{Mn}$ y $\mathrm{Zn}$.

Estos resultados concuerdan con lo reportado por Macedo y Jan (2008), quienes encontraron que a mayor concentración de aluminio en la solución nutritiva también hubo incrementos en las concentraciones de $\mathrm{Cu}$ y Fe, en tanto que Ramachandran et al. (2004) observaron que en presencia de aluminio la concentración de Mn se redujo. De acuerdo con Fageria y Carvalho (1982), el aluminio ejerce un efecto

Cuadro 1. Efecto del factor variedad, concentración de aluminio y de sus interacciones en la concentración de $\mathrm{Cu}, \mathrm{Fe}, \mathrm{Mn}$ y Zn en hojas de arroz.

\begin{tabular}{llcc}
\hline \multicolumn{1}{c}{ Catión } & \multicolumn{1}{c}{ Fuente de variación } & Grados de libertad & \multicolumn{2}{c}{ Significancia } \\
\hline $\mathrm{Cu}$ & Variedad & 2 & $* *$ \\
& Concentración & 3 & $* *$ \\
$\mathrm{Fe}$ & Variedad x Concentración & 6 & $*$ \\
& Variedad & 2 & $*$ \\
& Concentración & 3 & $\mathrm{~ns}$ \\
$\mathrm{Mn}$ & Variedad x Concentración & 6 & $*$ \\
& Variedad & 2 & $* *$ \\
$\mathrm{Zn}$ & Concentración & 3 & $* *$ \\
& Variedad x Concentración & 6 & $*$ \\
& Variedad & 2 & $* *$ \\
& Concentración & 3 & $*$ \\
& Variedad x Concentración & 6 & $*$ \\
\hline
\end{tabular}

ns: no significativo; ${ }^{*}=$ significativo a $5 \%$; $^{* *}$ = significativo a $1 \%$. 
Cuadro 1. Efecto de la interacción variedad x concentración de aluminio en la solución nutritiva sobre la concentración de $\mathrm{Cu}, \mathrm{Fe}, \mathrm{Mn}$ y Zn en hojas de arroz.

\begin{tabular}{|c|c|c|c|c|c|}
\hline \multirow{2}{*}{ Variedad } & \multirow{2}{*}{$\begin{array}{c}\text { Concentración de } \\
\mathrm{Al}(\mu \mathrm{M})\end{array}$} & \multicolumn{4}{|c|}{ Concentración micronutrimental $\left(\mathrm{g} \mathrm{kg}^{-1}\right.$ de materia seca) } \\
\hline & & $\mathrm{Cu}$ & $\mathrm{Fe}$ & $\mathrm{Mn}$ & $\mathrm{Zn}$ \\
\hline \multirow[t]{3}{*}{ 'Huimanguillo' } & 0 & $0.018 \mathrm{ef}$ & $0.573 \mathrm{~b}$ & $0.758 \mathrm{a}$ & $0.084 \mathrm{~cd}$ \\
\hline & 200 & $0.019 \mathrm{ef}$ & $0.718 \mathrm{ab}$ & $0.389 \mathrm{c}$ & $0.096 \mathrm{c}$ \\
\hline & 400 & $0.023 \mathrm{cdef}$ & $1.641 \mathrm{ab}$ & $0.304 \mathrm{~cd}$ & $0.134 \mathrm{ab}$ \\
\hline \multirow[t]{3}{*}{ 'Koshihikari' } & 0 & $0.016 \mathrm{f}$ & $0.758 \mathrm{ab}$ & $0.602 \mathrm{ab}$ & $0.062 \mathrm{~d}$ \\
\hline & 200 & $0.022 \mathrm{def}$ & $1.031 \mathrm{ab}$ & $0.395 \mathrm{c}$ & $0.107 \mathrm{bc}$ \\
\hline & 400 & $0.030 \mathrm{bcd}$ & $2.123 \mathrm{a}$ & $0.176 \mathrm{~d}$ & $0.135 \mathrm{ab}$ \\
\hline \multirow[t]{3}{*}{ 'Temporalero' } & 0 & 0.026 bcde & $1.879 \mathrm{ab}$ & $0.689 \mathrm{a}$ & $0.108 \mathrm{bc}$ \\
\hline & 200 & $0.035 \mathrm{~b}$ & $1.854 \mathrm{ab}$ & $0.745 \mathrm{a}$ & $0.157 \mathrm{a}$ \\
\hline & 400 & $0.046 \mathrm{a}$ & $1.879 \mathrm{ab}$ & $0.469 \mathrm{bc}$ & $0.163 \mathrm{a}$ \\
\hline \multirow[t]{3}{*}{ 'Tres Ríos’ } & 0 & $0.021 \mathrm{def}$ & $0.617 \mathrm{ab}$ & $0.424 \mathrm{bc}$ & $0.099 \mathrm{c}$ \\
\hline & 200 & $0.021 \mathrm{def}$ & $1.306 \mathrm{ab}$ & $0.377 \mathrm{c}$ & $0.109 \mathrm{bc}$ \\
\hline & 400 & $0.032 \mathrm{bc}$ & $1.820 \mathrm{ab}$ & $0.361 \mathrm{~cd}$ & $0.112 b c$ \\
\hline $\mathrm{DMSH}^{+}$ & & 0.010 & 1.539 & 0.192 & 0.034 \\
\hline
\end{tabular}

Medias con letras distintas en la misma columna indican diferencias estadísticas significativas (Tukey, 0.05$) .{ }^{\dagger} \mathrm{DMSH}=$ diferencia mínima significativa honesta.

Cuadro 3. Concentración de $\mathrm{Cu}, \mathrm{Fe}, \mathrm{Mn}$ y Zn en hojas de arroz sometidas a estrés por aluminio. I: Efecto del factor variedad.

\begin{tabular}{lcccc}
\hline \multirow{2}{*}{ Variedad } & \multicolumn{4}{c}{ Concentración micronutrimental $\left(\mathrm{g} \mathrm{kg}^{-1}\right.$ de materia seca $)$} \\
\cline { 2 - 5 } & $\mathrm{Cu}$ & $\mathrm{Fe}$ & $\mathrm{Mn}$ & $\mathrm{Zn}$ \\
\hline 'Huimanguillo' & $0.020 \mathrm{c}$ & $0.977 \mathrm{~b}$ & $0.484 \mathrm{~b}$ & $0.105 \mathrm{~b}$ \\
'Koshihikari' & $0.023 \mathrm{~b}$ & $1.247 \mathrm{ab}$ & $0.391 \mathrm{c}$ & $0.101 \mathrm{~b}$ \\
'Temporalero' & $0.036 \mathrm{a}$ & $1.871 \mathrm{a}$ & $0.634 \mathrm{a}$ & $0.143 \mathrm{a}$ \\
'Tres Ríos' & $0.025 \mathrm{bc}$ & $1.247 \mathrm{ab}$ & $0.387 \mathrm{c}$ & $0.107 \mathrm{~b}$ \\
$\mathrm{DMSH}^{\dagger}$ & 0.004 & 0.679 & 0.084 & 0.015 \\
\hline
\end{tabular}

Medias con letras distintas en la misma columna indican diferencias estadísticas significativas (Tukey, 0.05 ). ${ }^{`} \mathrm{DMSH}=$ diferencia mínima significativa honesta.

inhibitorio sobre la absorción de micronutrimentos en el siguiente orden: $\mathrm{Mn}>\mathrm{Zn}>\mathrm{Fe}>\mathrm{Cu}>\mathrm{B}$, lo cual también es coincidente con los resultados aquí mostrados. Sin embargo, Jan y Pettersson (1993) reportaron que a bajas concentraciones de $\mathrm{Al}(35,70$ y $140 \mu \mathrm{M} \mathrm{Al})$, las variedades BG35, DA14, y IR4 no mostraron efectos en las concentraciones de $\mathrm{Fe}, \mathrm{Zn}, \mathrm{Cu}$ ni Mn, lo cual demuestra diferentes mecanismos de respuesta entre los genotipos.

Tsutsui et al. (2012) reportaron que el gen que codifica la proteína transportadora de hierro 2 (OsIRT2; Os03g0667500) fue inducido en presencia de Al. Por su parte, Ishimaru et al. (2006) encontraron que la absorción de Fe es mediada por las proteínas OsIRT1 y OsIRT2 en arroz, aunque solo el gen OsIRT2 (pero no OsIRT1) es inducido por Al. Además, esta inducción en arroz parece ser única para la especie, dado que en Arabidopsis (Arabidopsis thaliana), maíz (Zea mays), trébol (Medicago truncatula) y trigo no se ha observado tal mecanismo de respuesta a aluminio.

El aluminio induce peroxidación y formación de especies reactivas de oxígeno, y en arroz se han identificado mecanismos antioxidantes que contrarrestan la toxicidad por $\mathrm{Al}$ (Meriga et al., 2004; Sharna y Dubey, 2007). Tsutsui et al. (2012) identificaron un gen con actividad de superóxido dismutasa (OsSOD) dependiente de Mn, lo que destaca la importancia de este metal en mecanismos antioxidantes detonados por el aluminio.

En cuanto a crecimiento, la mayor longitud de raíz (23.1 $\mathrm{cm})$ se presentó en la variedad 'Koshihikari', en plantas testigo $\left(0 \mu \mathrm{M} \mathrm{Al}^{3+}\right)$ (Figura 1), en tanto que la menor longitud $(13.6 \mathrm{~cm})$ se registró en la variedad 'Temporalero' tratada con $400 \mu \mathrm{M} \mathrm{Al}^{3+}$. Por su parte, la concentración de $200 \mu \mathrm{M} \mathrm{Al}^{3+}$ en solución causó una disminución ligera de 
Cuadro 4. Concentración de $\mathrm{Cu}, \mathrm{Fe}, \mathrm{Mn}$ y $\mathrm{Zn}$ en hojas de arroz sometidas a estrés por aluminio. II: Efecto del factor concentración de aluminio.

\begin{tabular}{lcccc}
\hline \multirow{2}{*}{$\begin{array}{c}\text { Concentración de } \mathrm{Al} \\
(\mu \mathrm{M})\end{array}$} & \multicolumn{4}{c}{ Concentración micronutrimental $\left(\mathrm{g} \mathrm{kg}^{-1}\right.$ de materia seca) } \\
\cline { 2 - 5 } & $0.020 \mathrm{c}$ & $\mathrm{Fe}$ & $\mathrm{Mn}$ & $\mathrm{Zn}$ \\
\hline 0 & $0.024 \mathrm{~b}$ & $0.957 \mathrm{~b}$ & $0.618 \mathrm{a}$ & $0.883 \mathrm{a}$ \\
200 & $0.033 \mathrm{a}$ & $1.227 \mathrm{~b}$ & $0.476 \mathrm{~b}$ & $0.217 \mathrm{~b}$ \\
400 & 0.003 & $1.866 \mathrm{a}$ & $0.327 \mathrm{c}$ & $0.136 \mathrm{c}$ \\
$\mathrm{DMSH}^{\dagger}$ & 0.533 & 0.066 & 0.012 \\
\hline
\end{tabular}

Medias con letras distintas en la misma columna indican diferencias estadísticas significativas (Tukey, 0.05). ${ }^{\dagger} \mathrm{DMSH}=$ diferencia mínima significativa honesta.

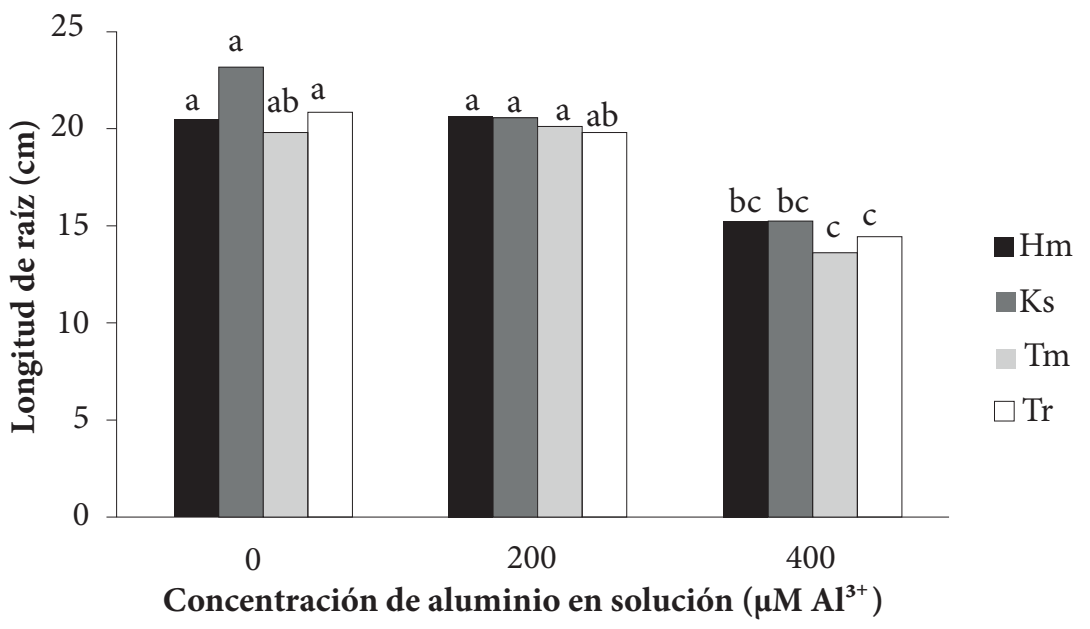

Figura 1. Longitud de raíz de cuatro variedades de arroz sometidas a estrés por aluminio durante cuatro semanas. Variedades: Hm: 'Huimanguillo'; Ks: 'Koshihikari'; Tm: 'Temporalero'; Tr: 'Tres Ríos'. Letras distintas sobre las columnas indican diferencias estadísticas significativas (Tukey, 0.05).

esta variable en la variedad 'Tres Ríos’

Estos resultados concuerdan con lo reportado por Goh y Lee (1999), quienes observaron que la inhibición del crecimiento de la raíz en plántulas de arroz depende de la concentración de $\mathrm{Al}$ en el medio, dado que las plantas absorben rápidamente este elemento.

La interacción del aluminio con los componentes de la membrana celular altera su permeabilidad, lo cual constituye uno de los principales factores responsables de la reducción del crecimiento de la raíz y de la planta (Peixoto et al., 2001). También existe una relación inversa entre la inhibición del crecimiento de la raíz y la acumulación de $\mathrm{Al}$ en la célula, porque el $\mathrm{Al}$ promueve la formación de especies reactivas de oxígeno (ROS) mediada por la peroxidación de lípidos. En especies vegetales que logran desarrollar mecanismos eficientes de destoxificación por $\mathrm{Al}$, la peroxidación es mitigada por medio de la activación del sistema antioxidante tanto enzimático como no enzimático (Meriga et al., 2004).
En comparación con el efecto observado en la concentración de macronutrimentos reportado por Marín-Garza et al. (2010), en donde se registró mayor tolerancia en la variedad 'Tres Ríos', en este estudio la variedad que más destaca es 'Temporalero', lo cual permite inferir que entre los genotipos de arroz hay diferentes niveles de importancia o efectos antagónicos del aluminio con los macro y los micronutrimentos.

Tsutsui et al. (2012) expusieron plantas de la variedad 'Koshihikari' (tolerante a $\mathrm{Al}$ ) y el mutante star1 (Sensitive to $\mathrm{Al}$ Rhizotoxicity 1 , sensible a $\mathrm{Al}$ ) a $20 \mathrm{mM} \mathrm{AlCl}_{3}$. Al aislar transcritos de células del ápice de la raíz para medir la expresión génica, encontraron que en el primer genotipo 213 genes mostraron inducción por $\mathrm{Al}$, en tanto que en el segundo fueron 2015 los genes inducidos. Al analizar los transcritos de células de la región basal de la raíz, observaron que para 'Koshihikari' hubo 126 genes inducidos por Al, mientras que para el mutante star1 fueron 2419 los genes inducidos por el metal. 
Estos resultados indican que los genes que responden a $\mathrm{Al}$ no son restrictivos del ápice de la raíz, sino que también hay en la región basal. Además del factor de transcripción ART1-regulated one (Yamaji et al., 2009), se encontraron nuevos mecanismos que relacionan la tolerancia del arroz a Al con la asimilación de $\mathrm{N}$ y con la síntesis de metabolitos secundarios, de pared celular y de etileno (Tsutsui et al., 2012), los cuales explican, al menos en parte, la alta tolerancia de esta especie al $\mathrm{Al}$, dado que especies sensibles a este metal no muestran inducción de tales mecanismos.

\section{CONCLUSIONES}

El aluminio afecta diferencialmente la acumulación de micronutrimentos en los diferentes genotipos de arroz analizados, y en general, al incrementarse la concentración de este metal en la solución nutritiva disminuyen las concentraciones de $\mathrm{Cu}$ y $\mathrm{Fe}$, pero aumentan las de $\mathrm{Mn}$ y $\mathrm{Zn}$, lo cual demuestra efectos antagónicos y sinérgicos, respectivamente. En todos los casos, la variedad 'Temporalero' logró mayor concentración de $\mathrm{Cu}, \mathrm{Fe}, \mathrm{Mn}$ y Fe en hojas, en comparación con las otras tres variedades evaluadas. Con relación a la longitud de raíz, a medida que aumentó el nivel de $\mathrm{Al}$ en la solución nutritiva esta variable se redujo, y las variedades más afectadas fueron 'Temporalero’ y 'Tres Ríos'.

\section{AGRADECIMIENTOS}

A la Línea Prioritaria de Investigación No. 5 Biotecnología Microbiana, Vegetal y Animal del Colegio de Postgraduados por las facilidades y los apoyos otorgados para este proyecto, así como al Consejo Nacional de Ciencia y Tecnología por la beca de postgrado otorgada a TMG. También se agradece a la Biól. Leticia Tavitas-Fuentes y al M. C. Leonardo Hernández-Aragón del INIFAP Zacatepec (México), por haber proporcionado los materiales de arroz analizados.

\section{BIBLIOGRAFÍA}

Alcántar G. G. y M. Sandoval V. (1999) Manual de Análisis Químico de Tejido Vegetal. Sociedad Mexicana de la Ciencia del Suelo A. C. Publicación Especial No. 10. Chapingo, México. 156 p.

Berg G., L. Eberl and A. Hartmann (2005) The rhizosphere as a reservoir for opportunistic human pathogenic bacteria. Environmental Microbiology 7:1673-1685.

Fageria N. K. and J. R. P. Carvalho (1982) Influence of aluminum nutrient solutions on chemical composition in upland rice cultivars. Plant and Soil 69:31-44.

Famoso A. N., R. T. Clark, J. E. Shaff, E. Craft, S. R. McCouch and L. V. Kochian (2010) Development of a novel aluminum tolerance phenotyping platform used for comparisons of cereal aluminum tolerance and investigations into rice aluminum tolerance mechanisms. Plant Physiology 153:1678-1691.

Goh C. H. and Y. Lee (1999) Aluminium uptake and aluminium-induced rapid root growth inhibition of rice seedlings. Journal of Plant Biology 42:151-158.

Ishimaru Y., M. Suzuki, T. Tsukamoto, K. Suzuki, M. Nakazono, T. Kobayashi,Y. Wada, S. Watanabe, S. Matsuhashi, M. Takahashi, H. Nakanishi, S. Mori and N. K. Nishizawa (2006) Rice plants take up iron as an $\mathrm{Fe}^{3+}$-phytosiderophore and as $\mathrm{Fe}^{2+}$. Plant Journal 45:335-346.

Jan F. and S. Pettersson (1993) Effects of low aluminium levels on growth and nutrient relations in three rice cultivars with different tolerances to aluminium. Journal of Plant Nutrition 16:359-372.

Macedo C. E. and V. V. S. Jan (2008) Effect of aluminum on mineral nutrition in rice cultivars differing in aluminum sensitivity. Engenheria Agricola e Ambiental 12:363-369.

Marín-Garza T., F. C. Gómez-Merino, L. I. Trejo-Téllez, A. MuñozOrozco, L. Tavitas-Fuentes, L. Hernández-Aragón and A. Santacruz-Varela (2010) Physiological and nutrimental responses of rice varieties to aluminium concentration. Revista Fitotecnia Mexicana 33:37-44.

Meriga B., K. B. Reddya, R. Raoa, L. A. Reddya and P. B. K. Kishora (2004) Aluminium-induced production of oxygen radicals, lipid peroxidation and DNA damage in seedlings of rice (Oryza sativa). Journal of Plant Physiology 161:63-68.

Peixoto P. H. P., J. Cambraia, R. Sant'Anna, P. Mosquim and M. A. Moreira (2001) Aluminum effects on fatty acid composition and lipid peroxidation of a purified plasma membrane fraction of root apices of two sorghum cultivars. Journal of Plant Nutrition 24:1061-1070.

Ramachandran V., D. U. Patel and S. C. Kumar (2004) Influence of aluminium on mineral nutrition of rice seedlings grown in solution culture. Indian Journal of Plant Physiology 9:348-353

SAS Institute (2011) SAS/STAT User's Guide. Ver. 9.3. SAS Institute, Inc. Cary, NC 8621 p. http://support.sas.com/documentation/cdl/ en/statug/63962/PDF/default/statug.pdf.

Sasaki T., Y. Yamamoto, B. Ezaki, M. Katsuhara, S. J. Ahn, P. R. Ryan, E. Delhaize and H. Matsumoto (2004) A wheat gene encoding an aluminum-activated malate transporter. Plant Journal 37:645-653.

Sharma P. and R. S. Dubey (2007) Involvement of oxidative stress and role of antioxidative defense system in growing rice seedlings exposed to toxic concentrations of aluminum. Plant Cell Reproduction 26:2027-2038.

Silva S. (2012) Aluminium toxicity targets in plants. Journal of Botany. doi: $10.1155 / 2012 / 219462$.

Steiner A. A. (1984) The universal nutrient solution. In: Proceedings of the 6th International Congress on Soilles Culture. pp:633-650.

Steiner A. A. and H. van Winden (1970) Recipe for ferric salts of ethylene diamine tetraacetic acid. Plant Physiology 46:862-863.

Trejo-Téllez L. I. and F. C. Gómez-Merino (2007) Physiological and biochemical responses of tobacco plants to aluminum concentrations under greenhouse conditions. Terra Latinoamericana 25:251-259.

Tsutsui T., N. Yamaji, C. F. Huang, R. Motoyama, Y. Nagamura and J. F. Ma (2012) Comparative genome-wide transcriptional analysis of Al-responsive genes reveals novel al tolerance mechanisms in rice. PLoS ONE 7:e48197. doi:10.1371/journal.pone.0048197.

Vardar F. and M. Ünal (2007) Aluminum toxicity and resistance in higher plants. Advances in Molecular Biology 1:1-12.

Watanabe T. and K. Okada (2005) Interactive effects of $\mathrm{Al}, \mathrm{Ca}$ and other cations on root elongation of rice cultivars under low $\mathrm{pH}$. Annals of Botany 95:379-385.

Yamaji N., C. F. Huang, S. Nagao, M. Yano, Y. Sato, Y. Nagamura and J. F. Ma (2009) A zinc finger transcription factor ART1 regulates multiple genes implicated in aluminum tolerance in rice. Plant Cell 21:3339-3349. 